\title{
Management of hepatitis B virus prophylaxis in patients treated with disease-modifying therapies for multiple sclerosis: a multicentric Italian retrospective study
}

\author{
Antonio Riccardo Buonomo ${ }^{1}$. Giulio Viceconte ${ }^{1}$ (D) Massimiliano Calabrese ${ }^{2}$ - Giovanna De Luca ${ }^{3}$. \\ Valentina Tomassini ${ }^{3} \cdot$ Paola Cavalla ${ }^{4}$. Giorgia Teresa Maniscalco ${ }^{5} \cdot$ Diana Ferraro $^{6} \cdot$ Viviana Nociti $^{7} \cdot$ Marta Radaelli $^{8}$. \\ Maria Chiara Buscarinu ${ }^{9}$. Damiano Paolicelli ${ }^{10}$. Alberto Gajofatto ${ }^{2}$. Pietro Annovazzi ${ }^{11} \cdot$ Federica Pinardi $^{12}$. \\ Massimiliano Di Filippo ${ }^{13}$. Cinzia Cordioli ${ }^{14}$ - Emanuela Zappulo ${ }^{1} \cdot$ Riccardo Scotto $^{1} \cdot$ Ivan Gentile $^{1}$. \\ Antonio Luca Spiezia ${ }^{15}$. Martina Petruzzo ${ }^{15}$. Marcello De Angelis ${ }^{15}$. Vincenzo Brescia Morra ${ }^{15}$. Claudio Solaro ${ }^{16}$. \\ Claudio Gasperini ${ }^{17}$. Eleonora Cocco ${ }^{18} \cdot$ Marcello Moccia $^{15} \cdot$ Roberta Lanzillo ${ }^{15}$ on behalf of Raising Italian \\ Researchers in Multiple Sclerosis (RIREMS) study group
}

Received: 9 December 2021 / Revised: 1 February 2022 / Accepted: 2 February 2022 / Published online: 14 February 2022

(c) The Author(s) 2022, corrected publication 2022

\begin{abstract}
Background Patients with multiple sclerosis (MS) often receive disease-modifying therapies (DMTs) that can expose them to reactivation of potential occult hepatitis B virus (HBV) infection (pOBI). We aimed to evaluate the MS Centers behavior regarding HBV screening and prophylaxis in a large cohort of MS patients receiving anti-CD20 or cladribine.

Methods Retrospective, multicentric study recruiting Italian MS patients treated with rituximab, ocrelizumab and cladribine. Results We included 931 MS patients from 15 centers. All but 38 patients performed a complete HBV screening. Patients' age $>50$ years was significantly associated with no history of vaccination and HBsAb titres $<100 \mathrm{mIU}$ at baseline $(p<0.001)$. No significant correlation was found between post-vaccination HBsAb titres and type of treatment $(p=0.5)$, pre-or posttherapy vaccination $(p=0.2)$ and number of previous DMTs $(p=0.2)$. Among pOBI patients $(n=53), 21$ received antiviral prophylaxis, while only 13 had HBV DNA monitoring and 19 patients neither monitored HBV DNA nor received prophylaxis. Conclusions Baseline HBV screening in patients receiving anti-CD20 and cladribine is a consolidated practice. Nonetheless, HBV vaccination coverage is still lacking in such population and age is a significant factor associated with low HBV protection. Rituximab, ocrelizumab and cladribine did not impair HBV vaccine response. Almost 35\% of pOBI patients fail to receive $\mathrm{HBVr}$ prevention. Management of $\mathrm{HBV}$ prophylaxis could be improved in MS patients and further prospective studies are needed to assess the effectiveness of prophylactic strategies in such patients.
\end{abstract}

Keywords Multiple sclerosis $\cdot$ Hepatitis B $\cdot$ Ocrelizumab $\cdot$ Rituximab $\cdot$ Cladribine $\cdot$ Vaccination

\section{Background}

Multiple sclerosis (MS) is a demyelinating immune-mediated disease of the central nervous system with an estimated prevalence of 30 cases per 100000 inhabitants globally and 176 cases per 100000 inhabitants in Italy, representing the most common cause of non-traumatic disability in young

Moccia Marcello and Lanzillo Roberta contributed equally.

Giulio Viceconte

giulio.viceconte@gmail.com

Extended author information available on the last page of the article adults [1, 2]. Disease-modifying therapies (DMTs) for MS have an immunomodulatory effect, reducing inflammation without depleting lymphocytes, or an immunosuppressive effect, as it occurs with preferential B-cells depleting nucleoside analogs (cladribine) or selective B-cell depleting monoclonal antibodies (rituximab, ocrelizumab) [3].

B-cell depleting therapies are associated with potential risks of viral infections. Hepatitis B virus (HBV) infection is the most common chronic viral infection, with estimated $30 \%$ of the world population having serological evidence of current or past infection [4]. Occult HBV infection (OBI) is defined as the presence of HBV DNA in the liver-with or without detectable serum HBV DNA-in the absence of 
detectable $\mathrm{HBsAg}$. OBI is defined potential (pOBI) if HBV DNA in liver cannot be ruled out, but patient have $\mathrm{HBcAb}$ and/or anti-HBs detectable without HBsAg [5].

As such, B-cell depleting therapies potentially hold the risk of pOBI reactivation, also leading to acute liver failure [6]. Risk of reactivation is well known in patients treated with rituximab for hematology conditions, reaching $40 \%$ in HBsAg positive patients and ranging from 2 to $23 \%$ in pOBI patients [7]. Conversely, in other settings in which anti$\mathrm{CD} 20$ are used, such as rheumatology, reactivation risk is estimated to be lower, since the underlining immunological asset is expected to be less compromised than haematologic conditions. For instance, in patients treated with rituximab for rheumatoid arthritis, the incidence of reactivation is estimated to be negligible and a baseline HBsAb titer $>100$ has been demonstrated to be protective [8]. Nonetheless, fatal cases of HBV reactivation have been reported in patients with neurological autoimmune disorders treated with antiCD20 [9, 10].

No definite evidence has been provided so far concerning the best strategy to prevent HBV reactivation among MS patients, although the European Society of Clinical Microbiology and Infectious Diseases recommends administering antiviral prophylaxis for $\mathrm{HBsAg}$ positive and pOBI patients that receive anti-CD20 therapies, regardless of the baseline condition [11]. There are no current guidelines specifically aimed at managing pOBI, but recommendations are derived from the international guidelines on general HBV management from the European Association for the Study of the Liver (EASL), and the American Association for the Study of Liver Diseases (AASLD) [6, 12]

As for cladribine, fewer data are available since $\mathrm{HBsAg}$ positive patients have been excluded from phase III trials for its use in MS and no data about participants' HBV serostatus have been reported, although anecdotal cases of HBV reactivation following cladribine administration for MS have been described [13].

Vaccination is the best strategy to prevent HBV infection. Although HBV vaccination is compulsory in Italy for all the newborns since 1991, according to the Italian viral hepatitis surveillance system (SEIEVA), the incidence of acute HBV infections in 2018 in Italy was still 0.4 cases $/ 100000$ people; young adults (35-55 years) are the most represented group and $7.6 \%$ of new infections are reported among individuals who received vaccination, although in most cases with an incorrect schedule [14]. Notably, it is well established that DMTs for MS, especially anti-CD20, can reduce the response to several vaccinations, but no data are available on HBV seroprevalence and response to HBV vaccination in such population [15]. The fact that the highest incidence of acute HBV infection occurs in the same age group in which the incidence of MS is higher, compels to better investigate and expand vaccination coverage of this population [16].

\section{Study aims}

We aimed to evaluate in a population of patients with MS and receiving ocrelizumab, rituximab or cladribine the following variables: (i) the proportion of MS patients who received complete HBV serological screening before commencing on ocrelizumab, rituximab or cladribine, defined as the availability in the clinical records of $\mathrm{HBsAg}, \mathrm{HBcAb}$ IgG and HBsAb before starting the DMT; (ii) the proportion of patients vaccinated for HBV before starting DMT therapy; (iii) the difference in pre- and post-vaccination HBsAb titers and its association with timing of vaccination, therapeutic history and age; (iv) choice of HBV reactivation prevention strategy (pre-emptive or antiviral prophylaxis) in pOBIs and its association with baseline HBsAb titers.

\section{Patients and methods}

This is a multicentric, retrospective, cross-sectional study involving 15 MS Centers across Italy. We retrospective examined the clinical records of patients with MS who are currently treated with ocrelizumab, rituximab or cladribine.

Clinical files from participating studies were reviewed by each involved center from July 2020 to January 2021, with cases being included from 2017 onwards.

Inclusion criteria were patients older than 18 years, affected by any form of MS, currently receiving ocrelizumab, rituximab or cladribine or that have received at least one dose of ocrelizumab, rituximab or cladribine in the last 6 months.

Exclusion criteria were concurrent DMTs or high-dose steroids ( $>20 \mathrm{mg} /$ daily of prednisone or equivalent); patients that have received plasma-exchange during life or intravenous immunoglobulins in the last 6 months.

The following data have been collected: demographic and clinical characteristics; HBV baseline serostatus; previous medication history for MS; history of HBV vaccination during lifetime; history of HBV vaccination during followup at MS Center; HBsAb titres at baseline and after HBV vaccination.

Patient's data were collected in an electronic database. Each MS Center was asked to send back the filled database. GV, ARB, MM, SR jointed and formatted the received data. No data were deleted from the original databases.

\section{Statistical analysis}

Categorical variables were confronted using bivariate logistic regression analysis; continuous variables were confronted to continuous variables using Spearman's Rho correlation 
and to categorical variables with Mann-Whitney $U$ test for dichotomous variables or with Kruskal-Wallis test for categorical variables with more than 2 values. A significance level of 0.05 was set for the interpretation of the results. Statistical analyses were performed using IBM SPSS Statistics version 27.

\section{Results}

\section{Study population}

We included 931 MS patients (median age: 47, IQR 38-54 years; females: $559,60 \%)$ treated with ocrelizumab $(n=738,79.3 \%)$, rituximab $(n=69,7.4 \%)$, or cladribine $(n=124,13.3 \%)$ from 15 Italian MS Centers. Follow-up was available for 747 patients $(15.9 \pm 9.1$ months). Demographics and clinical features are reported in Table 1.

\section{HBV screening}

All $(n=931)$ but 38 patients $(4 \%)$ had completed HBV screening before starting treatment. Of 38 patients with incomplete screening, 24 were treated with ocrelizumab, 13 with rituximab and 11 with cladribine. Among patients who received a complete screening $(n=893)$, no patients resulted positive to HBsAg. However, 310 patients (34\%) were positive to $\mathrm{HBsAb}$, of which 41 (4\%) also resulted in $\mathrm{HBc} A b-\mathrm{IgG}$ positive, as from the previous infection.

\section{Vaccination history and pOBI}

Among the $269 \mathrm{HBsAb}$-positive and HBcAb-IgG-negative patients, 134 (15\%) reported previous vaccination, while 6 reported no history of vaccination and 129 had vaccination history missing; 7 patients resulted in $\mathrm{HBcAb}-\mathrm{IgG}$ positive in the absence of HBsAb. Thus, 53 patients $(6 \%)$ were considered as pOBI (41 $\mathrm{HBsAb}+/ \mathrm{HBcAb}+; 6 \mathrm{HBsAb}+1$ $\mathrm{HBcAb}$ - not vaccinated; $6 \mathrm{HBs} \mathrm{Ab}-/ \mathrm{HBcAb}+)$. Only 184 out of $310 \mathrm{HBs} A b$ positive patients presented with tires $>100$ $\mathrm{mIU} / \mathrm{mL}$ (51.2\%). Detailed results of HBV screening are reported in Table 2 .

\section{Vaccination response}

Among 893 patients who received complete screening, quantitative $\mathrm{HBsAb}$ was available for 865 patients: among them, 681 presented with baseline HBsAb titer negative or $<100$ $\mathrm{mIU} / \mathrm{mL}$. Age $>50$ years was significantly associated with no history of vaccination and HBsAb titres $<100 \mathrm{mIU}$ at baseline $(p<0.001)$, while number of previous DMTs $>2$ $(p=0.8)$ was not (Table 3$)$.
Table 1 Demographics and clinical features of enrolled patients $(N=931)$

\begin{tabular}{lllll}
\hline & Ocrelizumab & Rituximab & Cladribine & Total \\
& $N=738$ & $N=69$ & $N=124$ & $N=931$ \\
\hline Age, years, median (IQR) & $47(39-55)$ & $51(42-59)$ & $39(29-47)$ & $47(38-54)$ \\
Female, $n(\%)$ & $434(58.8)$ & $49(71)$ & $85(68.5)$ & $559(60)$ \\
Treatment naïve, $n(\%)$ & $182(24.6)$ & $26(37.6)$ & $31(25)$ & $239(25)$ \\
Availability of follow-up, $\%)$ & $593(80.3)$ & $60(86.9)$ & $94(75.8)$ & $747(80)$ \\
Follow-up duration, months \pm SD & $16.9 \pm 8.9$ & $20.0 \pm 11.8$ & $8.8 \pm 5.8$ & $15.9 \pm 9.1$ \\
Previous DMT, $n(\%)$ & & & & \\
Alemtuzumab & $19(2.6)$ & $2(2.9)$ & $0(0)$ & \\
Azathioprine & $28(3.8)$ & $5(7.2)$ & $1(0.8)$ & \\
Cyclophosphamide & $14(1.9)$ & $2(2.9)$ & $0(0)$ & \\
Cladribine & $2(0.3)$ & $0(0)$ & $0(0)$ & \\
Daclizumab & $2(0.3)$ & $0(0)$ & $0(0)$ & \\
Dimethyl fumarate & $113(15.3)$ & $2(2.9)$ & $36(29)$ & \\
Fingolimod & $108(14.6)$ & $6(8.7)$ & $23(18.5)$ & \\
Glatiramer acetate & $51(6.9)$ & $2(2.9)$ & $7(5.6)$ & \\
Interferon beta & $51(6.9)$ & $6(8.7)$ & $16(12.9)$ & \\
Methotrexate & $3(0.4)$ & $2(2.9)$ & $0(0)$ & \\
Mitoxantrone & $1(0.1)$ & $4(5.8)$ & $0(0)$ & $7(5.6)$ \\
Natalizumab & $74(10)$ & $5(7.2)$ & $0(0)$ & \\
Rituximab & $51(6.9)$ & $0(0)$ & $0(0)$ & \\
Siponimod & $4(0.5)$ & $0(0)$ & $2(2.9)$ & \\
Teriflunomide & $46(6.2)$ & & & \\
\hline
\end{tabular}

$D M T$ disease-modifying therapy 
Table 2 HBV screening at baseline

\begin{tabular}{lllll}
\hline Patients with complete baseline screening & $\begin{array}{l}\text { Ocrelizumab } \\
N=714\end{array}$ & $\begin{array}{l}\text { Rituximab } \\
N=56\end{array}$ & $\begin{array}{l}\text { Cladribine } \\
N=113\end{array}$ & $\begin{array}{l}\text { Total } \\
N=893\end{array}$ \\
\hline HBsAg, positive (\%) & $0(0)$ & $0(0)$ & $0(0)$ & $0(0)$ \\
HBsAb positive-HBcAb negative, $n(\%)$ & $209(29)$ & $11(19)$ & $49(43)$ & $269(30)$ \\
Previous infection, $n(\%)$ & $33(5)$ & $7(12)$ & $1(0.8)$ & $41(4)$ \\
HBV vaccination & $112(15)$ & $2(3)$ & $20(17)$ & $134(15)$ \\
HBsAb $>100 \mathrm{mIU} / \mathrm{mL}, n(\%)$ & $154(21)$ & $6(1)$ & $24(21)$ & $184(20)$ \\
pOBI, $n(\%)$ & $38(5)$ & $9(16)$ & $6(5)$ & $53(6)$ \\
\hline
\end{tabular}

Previous infection: $\mathrm{HBs} A b$-positive, $\mathrm{HBcAb}-\mathrm{IgG}$-positive patients; $p O B I$ potential occult $\mathrm{HBV}$ infection

Table 3 HBV management strategies

\begin{tabular}{|c|c|c|c|c|c|}
\hline \multirow[t]{2}{*}{ Baseline HBsAb titres, $n(\%)$} & \multicolumn{5}{|c|}{$\begin{array}{l}\text { Patients with complete baseline screening } \\
N=893\end{array}$} \\
\hline & $\begin{array}{l}\text { Negative or }<100 \mathrm{mIU} / \mathrm{mL} \\
681 \text { (76) }\end{array}$ & $\begin{array}{l}>100 \mathrm{mIU} / \mathrm{mL} \\
184(20)\end{array}$ & OR & $95 \%$ CI & $P$ value \\
\hline \multicolumn{6}{|l|}{ Age, $n(\%)$} \\
\hline$\leq 50$ years & 406 (58) & 134 (74) & 2.01 & $1.4-2.9$ & $<0.001$ \\
\hline$>50$ years & $291(41)$ & $45(25)$ & & & \\
\hline \multicolumn{6}{|l|}{ Number of previous DMTs, $n(\%)$} \\
\hline$<1$ & $305(47)$ & $80(46)$ & & & \\
\hline$>1$ & $349(52)$ & $88(53)$ & 1.04 & $0.74-1.46$ & 0.8 \\
\hline Previous vaccination during lifetime, $n(\%)$ & $\begin{array}{l}\text { Vaccination } \\
134(15)\end{array}$ & $\begin{array}{l}\text { No vaccination } \\
509(56)\end{array}$ & & & \\
\hline \multicolumn{6}{|l|}{ Age, $n(\%)$} \\
\hline$\leq 50$ years & $116(86)$ & $242(47)$ & 7.1 & $4.2-12$ & $<0.001$ \\
\hline \multirow[t]{2}{*}{$>50$ years } & $18(13)$ & $267(52)$ & & & \\
\hline & $\begin{array}{l}\text { pOBI patients } \\
n=53(5.9 \%)\end{array}$ & & & & \\
\hline Baseline HBsAb titres, $n(\%)$ & $\begin{array}{l}\text { Negative or }<100 \mathrm{mIU} / \mathrm{mL} \\
13 \text { (24) }\end{array}$ & $\begin{array}{l}>100 \mathrm{mIU} / \mathrm{mL} \\
40(75)\end{array}$ & & & \\
\hline Antiviral prophylaxis & $6(46)$ & $15(37)$ & 1.1 & $0.7-1.3$ & 0.6 \\
\hline HBV DNA monitoring & $0(0)$ & $13(32)$ & 1 & $0.67-1.48$ & 0.1 \\
\hline \multirow[t]{2}{*}{ No intervention } & $7(53)$ & $12(30)$ & 1.31 & $0.74-2.33$ & 0.3 \\
\hline & \multicolumn{2}{|c|}{$\begin{array}{l}\text { Pre- or post-therapy vaccinated patients } \\
n=86(9.6 \%)\end{array}$} & & & \\
\hline Post-vaccination titres, mIU/mL, median (IQR) & \multicolumn{2}{|c|}{$61.5(1.25-168.5)$} & & & \\
\hline Number of previous DMTs, median (IQR) & \multicolumn{2}{|l|}{$2(1-2)$} & & & 0.2 \\
\hline \multicolumn{3}{|l|}{ Type of treatment } & & & 0.5 \\
\hline Ocrelizumab, $n(\%)$ & \multicolumn{2}{|l|}{$66(76.7)$} & & & \\
\hline Rituximab, $n(\%)$ & \multicolumn{2}{|l|}{$3(3.4)$} & & & \\
\hline Cladribine, $n(\%)$ & \multicolumn{2}{|l|}{$14(1.5)$} & & & \\
\hline \multicolumn{3}{|l|}{ Timing of vaccination } & & & 0.2 \\
\hline Pre-therapy & \multicolumn{2}{|l|}{$81(94)$} & & & \\
\hline Time to follow-up, months, mean $\pm \mathrm{SD}$ & \multicolumn{2}{|l|}{$5.4 \pm 3.6$} & & & \\
\hline Post-vaccination titres, mIU/mL, median (IQR) & \multicolumn{2}{|l|}{$61.5(1.25-191.5)$} & & & \\
\hline Post-therapy & \multicolumn{2}{|l|}{$5(5.8)$} & & & \\
\hline Time to follow-up, months, mean \pm SD & \multicolumn{2}{|l|}{$5.9 \pm 3.2$} & & & \\
\hline Post-vaccination titres, mIU/mL, median (IQR) & \multicolumn{2}{|l|}{$205(2-205)$} & & & \\
\hline
\end{tabular}

$D M T$ disease-modifying therapy 
Globally, 81 patients received $\mathrm{HBV}$ vaccination before commencing on DMT (ocrelizumab $=64$; rituximab $=3$; cladribine $=14$ ). Among them, only 15 (ocrelizumab $=13$; rituximab $=1$; cladribine $=1$ ) had $\mathrm{HBs} \mathrm{Ab}$ titer available, which was performed after a mean of $5.4 \pm 3.6$ months after DMT's administration, and the median titer was $61.5 \mathrm{mIU} /$ mL (IQR 1.25-191.5).

In addition, 5 patients received $\mathrm{HBV}$ vaccination after commencing DMTs (ocrelizumab $=4$; rituximab $=1$ ); 4 of them had HBsAb titer checked after a mean time of $5.9 \pm 3.2$ months (ocrelizumab $=3$; rituximab $=1$ ), with a median titer of $205 \mathrm{mIU} / \mathrm{mL}$ (IQR 2-205), and only 1 patient treated with ocrelizumab remained below protective levels. No significant correlation has been found between post-vaccination HBsAb titres $(n=19)$ and type of treatment $(p=0.5)$; pre-or post-therapy vaccination $(p=0.2)$ and number of previous DMTs $(p=0.2)$.

\section{Prevention strategies}

Among patients potentially at risk of $\mathrm{HBV}$ reactivation (pOBI, $n=53$ ), 20 received antiviral prophylaxis with lamivudine and 1 with entecavir, while only 13 had HBV DNA monitoring (every 3-6 months, pre-emptive strategy) in the absence of antiviral prophylaxis. $89,8 \%$ of pOBI patients were on treatment with anti-CD20 (ocrelizumab or rituximab). Notably, pre-emptive strategy was adopted in all patients with $\mathrm{HBsAb}>100 \mathrm{mIU} / \mathrm{mL}$. Conversely, 19 patients neither monitored HBV-DNA nor received antiviral prophylaxis. In detail, $53 \%$ of patients with negative or $<100 \mathrm{mIU} /$ $\mathrm{mL} \mathrm{HBsAb}$ and $30 \%$ of patients with $\mathrm{HBsAb}>100 \mathrm{mIU} / \mathrm{mL}$ did not receive neither prophylaxis nor monitoring. Antiviral prophylaxis was more often prescribed in patients with $\mathrm{HBsAb}<100 \mathrm{mIU} / \mathrm{mL}$. Nonetheless, no significant association was found between the choice of HBV reactivation prevention strategy and HBsAb titres < or > than $100 \mathrm{mIU} /$ $\mathrm{mL}$. However, no cases of $\mathrm{HBV}$ reactivation were detected in both groups.

\section{Discussion}

In this multicentric study, we showed that patients affected by MS treated with B cells depleting DMTs are very likely to receive complete HBV screening before commencing treatment in clinical practice. Nonetheless, more than half of patients older than 50 years reported no HBV vaccination or had vaccination history missing, and a high proportion of them were HBsAb negative or with a titer lower than 100 $\mathrm{UI} / \mathrm{mL}$. Thus, vaccination coverage is still lacking in a large part of Italian MS population, increasing the risk of de-novo severe forms of $\mathrm{HBV}$ infection, considering the exposure to immunodepleting DMTs. In particular, $24 \%$ of pOBI patients resulted to have higher risk of $\mathrm{HBV}$ reactivation, because of negative or $<100 \mathrm{mIU} / \mathrm{mL} \mathrm{HBs} A b$ titer. Even though no definite recommendations exist for the choice of the best HBV reactivation prevention in such a population, we observed a trend to prescribing antiviral prophylaxis in patients with negative or $<100 \mathrm{mIU} / \mathrm{mL} \mathrm{HBs} A b$ more often than in patients with $\mathrm{HBs} \mathrm{Ab}>100 \mathrm{mIU} / \mathrm{mL}$, even if the difference was not statistically significant.

No HBV reactivation was reported over 15 months of follow-up, inthis small number of pOBI patients. It is noteworthy, however, from previous studies including patients receiving monoclonal antibodies for rheumatic diseases, that pOBI patients with negative $\mathrm{HBsAb}$ are at higher risk of reactivation, while $\mathrm{HBs} \mathrm{Ab}>100 \mathrm{mIU} / \mathrm{mL}$ could be considered a protective factor for HBV reactivation [8, 17].

Thus, considering the results of our study, antiviral prophylaxis could be prescribed in MS patients receiving B-cell depleting agents with pOBI and HBsAb negative or $<100$ $\mathrm{mIU} / \mathrm{mL}$, while in pOBI patients with $\mathrm{HBsAb}>100 \mathrm{mIU} /$ mL HBV DNA monitoring without prophylaxis could be a valid and cost-effective measure. However, further studies are required to confirm these observational findings. Nonetheless, the fact that $35 \%$ of pOBI patients failed to receive both preventive measures (prophylaxis and monitoring), highlights the urgency of implementing a jointed protocol for HBV reactivation prevention among MS Centers, with a collaboration between neurologists and infectious diseases' specialists.

Together with the screening and prophylaxis approach, vaccination remains one of the cornerstones in HBV prevention. Among MS patients enrolled in our cohort, a trend towards reduction of $\mathrm{HBs} \mathrm{Ab}$ titres was observed in patients receiving ocrelizumab and in those who got vaccinated before starting the DMT, compared to those who were vaccinated after the start of the therapy, although the difference was not statistically significant. Since data on vaccination schedules and on dates of administration and HBsAb titres determination were too scarce, this difference can be imputable to different timing of $\mathrm{HBsAb}$ determination in the two groups.

Nonetheless, a reduction in $\mathrm{HBsAb}$ titres even below protective level is expected in patients receiving B-cell depleting agents, thus requiring frequent $\mathrm{HBs} \mathrm{Ab}$ monitoring in pOBI patients with initial titres $>100 \mathrm{mIU} / \mathrm{mL}$, especially when HBV reactivation prevention is managed with preemptive strategy. The opportunity to start antiviral prophylaxis, indeed, should be reconsidered on the basis of the point by point $\mathrm{HBs} \mathrm{Ab}$ determination, rather than baseline HBsAb [8, 17].

Although Italy has one of the highest prevalence of HBV infection in Europe, despite its reduction following compulsory HBV vaccination for all the newborns after 1991, we still do not know the prevalence of MS 
patients with chronic HBV infection or pOBI in real life since no studies have ever been conducted so far. Indeed, in the majority of clinical trials on non anti-CD20 DMTs, screening for HBV was not performed or was only focused on $\mathrm{HBsAg}$, while in phase 3 trials of ocrelizumab, pOBI patients were screened and monitored with HBV DNA every 12 weeks while on treatment, but the proportion of such patients, as well as the proportion of virological or clinical reactivations, were not reported by the authors [18-20]. The results of this study, even if derived from an Italian real-life cohort, could be generalized for other countries, even if we expect to find higher or lower percentage of pOBI and chronically infected patients depending of historical HBV prevalence (during the previous 30-40 years) according to the geographic areas (higher in Mediterranean area and Asia, lower in North America and North Europe), and to vaccination coverages. Thus, the urge of pOBI identification and prophylaxis could be variable according to these considerations.

\section{Conclusions}

To the best of our knowledge, this is the first study evaluating HBV serostatus, vaccination and prophylaxis in a population of MS patients receiving B-cell suppressant DMTs The main limitation of this study is the retrospective design, the scarcity of data about HBsAb titres and the incomplete follow-up. Further prospective studies are needed to define the best follow-up strategy for pOBI MS patients. From this study, we can conclude that a HBV vaccination campaign is needed to target our largely unvaccinated MS population. In particular, we hope that the results of this study will raise the awareness to actively vaccinate all patients diagnosed with MS, considering the possible necessity of using immunosuppressive DMTs in their disease course over time. In the next future, our study group is considering an active vaccination strategy for $\mathrm{HBV}$ in MS patients, and will evaluate prospectively the effectiveness of such campaign and its efficacy in long term in MS patients treated with DMTs.

\begin{abstract}
Author contributions BAR: conceptualization and supervision; VG: conceptualization and writing original draft; CM: investigation; DLG: investigation; TV: investigation; CP: investigation; MGT: investigation; FD: investigation; NV: investigation; RM: investigation; BMC: investigation; PD: investigation; GA: investigation; AP: investigation; PF: investigation; DFM: investigation; CC: investigation; ZE: supervision and validation; SR: formal analysis; GI: supervision; SA: investigation; PM: investigation; DAM: investigation; BMV: writing—review editing; SC: investigation; $\mathrm{GC}$ : investigation; $\mathrm{CE}$ : investigation; $\mathrm{MM}$ : supervision, review and editing; LR: supervision, review and editing.
\end{abstract}

Funding No funding source has been used.

\section{Declarations}

Conflicts of interest The authors have no competing interests to disclose.

Ethical standards The study was conducted according to 1964 Declaration of Helsinki and its later amendments. Approval from ethical committee was waived because of the retrospective nature of the study.

Open Access This article is licensed under a Creative Commons Attribution 4.0 International License, which permits use, sharing, adaptation, distribution and reproduction in any medium or format, as long as you give appropriate credit to the original author(s) and the source, provide a link to the Creative Commons licence, and indicate if changes were made. The images or other third party material in this article are included in the article's Creative Commons licence, unless indicated otherwise in a credit line to the material. If material is not included in the article's Creative Commons licence and your intended use is not permitted by statutory regulation or exceeds the permitted use, you will need to obtain permission directly from the copyright holder. To view a copy of this licence, visit http://creativecommons.org/licenses/by/4.0/.

\section{References}

1. Wallin MT, Culpepper WJ, Nichols E et al (2019) Global, regional, and national burden of multiple sclerosis 1990-2016: a systematic analysis for the Global Burden of Disease Study 2016. Lancet Neurol 18:269-285. https://doi.org/10.1016/S14744422(18)30443-5

2. Battaglia MA, Bezzini D (2016) (2016) Estimated prevalence of multiple sclerosis in Italy in 2015. Neurol Sci 383(38):473-479

3. Adamczyk-Sowa M, Mado H, Kubicka-Bączyk K et al (2021) SARS-CoV-2/COVID-19 in multiple sclerosis patients receiving disease-modifying therapy. Clin Neurol Neurosurg 201:106451. https://doi.org/10.1016/j.clineuro.2020.106451

4. Trépo C, Chan HLY, Lok A (2014) Hepatitis B virus infection. Lancet 384:2053-2063

5. Raimondo G, Allain J-P, Brunetto MR et al (2008) Statements from the Taormina expert meeting on occult hepatitis B virus infection. J Hepatol 49:652-657. https://doi.org/10.1016/j.jhep. 2008.07.014

6. EASL 2017 Clinical Practice Guidelines on the management of hepatitis B virus infection $\mathrm{q}$

7. Viganò M, Serra G, Casella G et al (2016) Reactivation of hepatitis B virus during targeted therapies for cancer and immunemediated disorders. Expert Opin Biol Ther 16:917-926

8. Chen Y, Chen H, Huang W et al (2019) Reactivation of hepatitis B virus infection following rituximab treatment in $\mathrm{HBsAg}$-negative, $\mathrm{HBcAb}$-positive rheumatoid arthritis patients: a long-term, realworld observation. Int J Rheum Dis 22:1756-1785. https://doi. org/10.1111/1756-185X.13582

9. Ciardi MR, Iannetta M, Zingaropoli MA et al (2019) Reactivation of hepatitis B virus with immune-escape mutations after ocrelizumab treatment for multiple sclerosis. Open Forum Infect Dis 6:1-3. https://doi.org/10.1093/ofid/ofy356

10. Buonomo AR, Scotto R, Coppola C et al (2020) Direct acting antivirals treatment for hepatitis $\mathrm{C}$ virus infection does not increase the incidence of de novo hepatocellular carcinoma occurrence: Results from an Italian real-life cohort (LINA cohort). Med (United States). https://doi.org/10.1097/MD.0000000000018948

11. Mikulska M, Lanini S, Gudiol C et al (2018) ESCMID Study Group for Infections in Compromised Hosts (ESGICH) Consensus 
Document on the safety of targeted and biological therapies: an infectious diseases perspective (Agents targeting lymphoid cells surface antigens [I]: CD19, CD20 and CD52). Clin Microbiol Infect 24:S71-S82

12. Terrault NA, Lok ASF, McMahon BJ et al (2018) Update on prevention, diagnosis, and treatment of chronic hepatitis B: AASLD 2018 hepatitis B guidance. Hepatology 67:1560-1599. https://doi. org/10.1002/hep.29800

13. Busuttil DP, Chasty RC, Fraser M et al (1996) Delayed reactivation of hepatitis B infection after cladribine [8]. Lancet 348:129

14. Bollettino Seieva. https://www.epicentro.iss.it/epatite/BollettinoSeieva. Accessed 14 Feb 2021

15. Ciotti JR, Valtcheva MV, Cross AH (2020) Effects of MS diseasemodifying therapies on responses to vaccinations: a review. Mult Scler Relat Disord 45:102439. https://doi.org/10.1016/j.msard. 2020.102439

16. Goodin DS (2014) The epidemiology of multiple sclerosis insights to disease pathogenesis. Handbook of Clinical Neurology. Elsevier, Amsterdam, pp 231-266
17. Tien YC, Yen HH, Li CF et al (2018) Changes in hepatitis B virus surface antibody titer and risk of hepatitis $\mathrm{B}$ reactivation in $\mathrm{HBs} A$-negative/HBcAb-positive patients undergoing biologic therapy for rheumatic diseases: A prospective cohort study. Arthritis Res Ther 20:246. https://doi.org/10.1186/s13075-018-1748-z

18. Hauser SL, Bar-Or A, Comi G et al (2017) Ocrelizumab versus interferon beta-1a in relapsing multiple sclerosis. N Engl J Med 376:221-234. https://doi.org/10.1056/NEJMOA1601277/SUPPL_ FILE/NEJMOA1601277_DISCLOSURES.PDF

19. Riederer F (2017) Ocrelizumab versus placebo in primary progressive multiple sclerosis. J fur Neurol Neurochir und Psychiatr 18:30-31. https://doi.org/10.1056/NEJMOA1606468/SUPPL_ FILE/NEJMOA1606468_DISCLOSURES.PDF

20. Epstein DJ, Dunn J, Deresinski S (2018) Infectious complications of multiple sclerosis therapies: implications for screening, prophylaxis, and management. Open Forum Infect Dis. https://doi.org/ 10.1093/OFID/OFY174

\title{
Authors and Affiliations
}

\author{
Antonio Riccardo Buonomo ${ }^{1}$. Giulio Viceconte ${ }^{1}$ (D) Massimiliano Calabrese ${ }^{2}$ - Giovanna De Luca ${ }^{3}$. \\ Valentina Tomassini ${ }^{3}$. Paola Cavalla ${ }^{4}$. Giorgia Teresa Maniscalco ${ }^{5}$. Diana Ferraro ${ }^{6} \cdot$ Viviana Nociti $^{7} \cdot$ Marta Radaelli $^{8}$. \\ Maria Chiara Buscarinu ${ }^{9}$. Damiano Paolicelli ${ }^{10}$. Alberto Gajofatto ${ }^{2}$. Pietro Annovazzi ${ }^{11}$. Federica Pinardi ${ }^{12}$. \\ Massimiliano Di Filippo ${ }^{13}$. Cinzia Cordioli ${ }^{14}$. Emanuela Zappulo ${ }^{1} \cdot$ Riccardo Scotto $^{1} \cdot$ Ivan Gentile ${ }^{1}$. \\ Antonio Luca Spiezia ${ }^{15}$. Martina Petruzzo ${ }^{15}$. Marcello De Angelis ${ }^{15}$. Vincenzo Brescia Morra ${ }^{15}$. Claudio Solaro ${ }^{16}$. \\ Claudio Gasperini ${ }^{17}$. Eleonora Cocco ${ }^{18} \cdot$ Marcello Moccia $^{15} \cdot$ Roberta Lanzillo $^{15}$ on behalf of Raising Italian \\ Researchers in Multiple Sclerosis (RIREMS) study group
}

1 Department of Clinical Medicine and Surgery-Section of Infectious Diseases, University of Naples "Federico II", Via Sergio Pansini 5, 80130 Naples, Italy

2 Department of Neuroscience, Biomedicine and Movement, The Multiple Sclerosis Center of the University Hospital of Verona, Verona, Italy

3 Institute for Advanced Biomedical Technologies (ITAB), Department of Neurosciences, Imaging and Clinical Sciences, University "G. d'Annunzio" of Chieti-Pescara, Chieti, Italy

4 Department of Neuroscience and Mental Health-City of Health and Science University, Hospital of Torino, Multiple Sclerosis Center, Turin, Italy

5 Multiple Sclerosis Center, “A. Cardarelli” Hospital, Naples, Italy

6 Department of Biomedical, Metabolic and Neurosciences, University of Modena and Reggio Emilia, Modena, Italy

7 Institute of Neurology, Fondazione Policlinico Universitario 'A. Gemelli' IRCCS, Catholic University of Sacred Heart, Rome, Italy

8 Department of Neurology and Multiple Sclerosis Center, ASST Papa Giovanni XXIII, Bergamo, Italy
9 Department of Neuroscience, Mental Health and Sensory Organs, Sapienza University, S. Andrea Hospital Site, Rome, Italy

10 Department of Basic Medical Sciences, Neuroscience and Sense Organs, University of Bari "Aldo Moro”, Bari, Italy

11 Multiple Sclerosis Center, ASST Valle Olona-Gallarate Hospital, Gallarate, VA, Italy

12 UOSI Multiple Sclerosis Rehabilitation, IRCCS, Bologna, Italy

13 Section of Neurology, Department of Medicine and Surgery, University of Perugia, Perugia, Italy

14 Multiple Sclerosis Center, Montichiari Hospital, ASST Spedali Civili Di Brescia, Brescia, Italy

15 Department of Neurosciences, Reproductive and Odontostomatological Sciences, University of Naples "Federico II", Naples, Italy

16 CRRF Mons. Luigi Novarese, Moncrivello, VC, Italy

17 Department of Neuroscience, San-Camillo Forlanini Hospital, Rome, Italy

18 Multiple Sclerosis Center, Binaghi Hospital, Azienda Tutela Della Salute (ATS) Sardegna, Cagliari, Italy 